\title{
DESENVOLVIMENTO E ANÁLISE SENSORIAL DE SOBREMESAS LÁCTEAS SABOR CHOCOLATE ENRIQUECIDAS COM AMÊNDOA DO BARU (Dipteryx Alata Vogel)
}

\author{
P. N. CRUZ ${ }^{1}$, C. B. OLIVEIRA ${ }^{2}$ e P. B. PERTUZATTI ${ }^{3}$ \\ ${ }^{1}$ Universidade Federal de Santa Catarina, Departamento de Engenharia de Alimentos \\ ${ }^{2}$ Universidade Federal de Mato Grosso, Departamento de Engenharia Alimentos \\ ${ }^{2}$ Universidade Federal de Mato Grosso, Departamento de Engenharia Alimentos \\ E-mail para contato: pollyannaicm@hotmail.com
}

\begin{abstract}
RESUMO - No trabalho foram desenvolvidas formulações de sobremesas lácteas de chocolate e baru utilizando um planejamento experimental com um delineamento composto central rotacional, incluindo 4 pontos axiais e 3 repetições no ponto central e realizada a análise sensorial descritiva quantitativa, teste de aceitação e intenção de compra. Na análise descritiva quantitativa foram escolhidos doze termos descritores. Os julgadores participaram de seis sessões de treinamento e foram selecionados somente os que obtiveram valores de Famostra significativo $(\mathrm{p} \leq 0,50)$ e Frepetição não significativos $(\mathrm{p} \geq 0,05)$. Na análise de aceitação e intenção de compra todas as amostras obtiveram boa aceitação, mas a formulação com $14 \%$ de chocolate e $7 \%$ de baru obteve maior média, significando uma maior preferência dos provadores.
\end{abstract}

\section{INTRODUÇÃO}

As sobremesas lácteas prontas para comer, com vida de prateleira média, apresentaram importante crescimento nas últimas décadas. O desenvolvimento dessas sobremesas com a utilização da castanha do baru pode constituir uma alternativa tecnológica para agregar valor funcional as mesmas (LIMA et al.; 2010). A análise sensorial é um campo muito importante na indústria de alimentos, pois contribui direta ou indiretamente para inúmeras atividades, como desenvolvimento de novos produtos, controle de qualidade, reformulação e redução de custos de produtos, relações entre condições de processo, ingredientes, aspectos analíticos e sensoriais (STONE, BLEIBAUM e THOMAS, 2012). Este trabalho teve por objetivo o desenvolvimento de formulações de sobremesas lácteas de chocolate e amêndoa do baru, através de um planejamento experimental $2^{2}$, tendo como variável resposta a avaliação sensorial pelo métodoADQ (Análise descritiva quantitativa), teste de aceitação e intenção de compra.

\section{MATERIAL E MÉTODOS}


As formulações utilizadas neste trabalho foram compostas pelos seguintes ingredientes: leite $(43 \%)$, açúcar $(18 \%)$, creme de leite $(17 \%)$, goma guar $(0,5 \%)$, sorbato de potássio $(0,08 \%)$, chocolate, amêndoa do baru e água que foi utilizada como quantidade suficiente para completar $100 \%$ da formulação. Após a adição dos ingredientes foi realizada a agitação para obter a correta distribuição dos componentes da massa, em seguida a massa foi submetida a aquecimento durante 6 minutos. Posteriormente, acondicionadas em embalagens de vidro previamente esterilizadas e em seguida, elas foram resfriadas à $4{ }^{\circ} \mathrm{C}$ e mantidas refrigeradas, durante $\mathrm{o}$ decorrer das análises.

Para a obtenção das diferentes formulações das sobremesas foi utilizado um delineamento fatorial completo $2^{2}$ composto, perfazendo onze ensaios de acordo com a Tabela 1 .

Tabela 1 - Variáveis e níveis do planejamento experimental completo $2^{2}$

\begin{tabular}{lccccc}
\hline & $-\alpha$ & \multicolumn{4}{c}{ Níveis codificados } \\
\cline { 2 - 6 } & $-1,41$ & -1 & 0 & +1 & $+1,41$ \\
\cline { 2 - 6 } Variáveis & \multicolumn{5}{c}{ Níveis reais dos ingredientes } \\
Independentes & \multicolumn{5}{c}{. } \\
\cline { 2 - 6 } Chocolate (\%) & 4 & 5,45 & 9 & 12,55 & 14 \\
Baru (\%) & 0 & 2,04 & 7 & 11,96 & 14 \\
\hline
\end{tabular}

As análises sensoriais realizadas nas sobremesas lácteas de chocolate e baru foram a análise descritiva quantitativa, análise de aceitação e intenção de compra.

Primeiramente foram pré-selecionados trinta e quatro indivíduos e na fase de seleção e treinamento dos julgadores foram realizados os testes de comparação pareada e teste triangular. Os candidatos pré-selecionados fizeram o levantamento dos termos descritores e com eles foi elaborada a ficha de avaliação com escalas não estruturadas de 9 centímetros ancoradas nos pontos extremos à esquerda pelo termo "fraco", ou "nenhum" e à direita "forte" ou "muito".

Após o levantamento dos atributos sensoriais, houve um treinamento com as referências durante seis sessões , para que fosse possível a formação da memória sensorial.

Foram selecionados os candidatos com base no poder de discriminação entre as amostras, repetibilidade e concordância com a equipe (DAMÁSIO e COSTELL, 1991), verificadas através de análise de variância de dois fatores (amostra e repetição) para cada provador em relação a cada atributo. Os resultados foram avaliados por Análise de Variância e um teste de média, o Tukey para comparação das amostras. Os resultados foram expressos pelo gráfico aranha.

No teste de aceitação foi utilizado uma escala hedônica estruturada de 9 pontos, variando de "'gostei extremamente' (9) a "'desgostei extremamente" (1) e a intenção de compra do consumidor, foi avaliada através de uma escala de 5 pontos variando de "certamente compraria" (5) a "'certamente não compraria', (1).

\section{RESULTADOS E DISCUSSÃO}

\subsection{Análise descritiva Quantitativa}


Para a pré-seleção dos provadores trinta e quatro consumidores de sobremesa láctea realizaram o teste pareado e triangular e somente oito provadores foram selecionados para participar da Análise Descritiva Quantitativa.

Os provadores selecionados geraram os seguintes termos descritores que encontram-se listados na tabela 2.

Tabela 2. Definições e Referências para os termos descritores geradas pela equipe sensorial descritiva

\begin{tabular}{|c|c|c|}
\hline Termo descritor & Definição & Referência \\
\hline \multicolumn{3}{|l|}{ Aparência } \\
\hline & & Fraco: Achocolatado Muky \\
\hline Cor marrom & Cor característica do produto & $\begin{array}{l}\text { Forte: Barra de chocolate com } \\
80 \% \text { de cacau }\end{array}$ \\
\hline \multirow[t]{3}{*}{ Presença de partículas } & \multirow{3}{*}{$\begin{array}{c}\text { Presença da castanha de baru que é } \\
\text { caracterizada por pontos pretos } \\
\text { (casca) }\end{array}$} & $\begin{array}{l}\text { Nenhuma: Sobremesa láctea } \\
\text { marca danete }\end{array}$ \\
\hline & & $\begin{array}{l}\text { Forte: Solução de leite c/ } 15 \% \text { de } \\
\text { baru }\end{array}$ \\
\hline & & Fraco: Bebida láctea Paulista \\
\hline Viscosidade & $\begin{array}{l}\text { Propriedade de resistência ao } \\
\text { escoamento }\end{array}$ & $\begin{array}{l}\text { Forte: Creme de avelã da marca } \\
\text { Nutella armazenada na geladeira }\end{array}$ \\
\hline \multirow{2}{*}{ Brilho } & \multirow[t]{2}{*}{$\begin{array}{l}\text { Capacidade da superfície do } \\
\text { produto de refletir a luz }\end{array}$} & $\begin{array}{l}\text { Fraco: Café em pó da marca } \\
\text { Nescafé }\end{array}$ \\
\hline & & Forte: Açucar derretido \\
\hline \multicolumn{3}{|l|}{ Aroma } \\
\hline \multirow[b]{2}{*}{ Chocolate } & \multirow[b]{2}{*}{$\begin{array}{c}\text { Aroma característico associado ao } \\
\text { chocolate }\end{array}$} & Fraco: Achocolatado Muky \\
\hline & & $\begin{array}{l}\text { Forte: Chocolate com } 50 \% \text { de } \\
\text { Cacau }\end{array}$ \\
\hline \multirow{2}{*}{ Amêndoa } & \multirow{2}{*}{$\begin{array}{c}\text { Aroma característico associado à } \\
\text { amêndoa }\end{array}$} & $\begin{array}{l}\text { Fraco: Mistura } 5 \% \text { de castanha } \\
\text { com } 95 \% \text { de chocolate }\end{array}$ \\
\hline & & Forte: Castanha de baru triturada \\
\hline \multicolumn{3}{|l|}{ Sabor } \\
\hline Amargo & \multirow[t]{2}{*}{$\begin{array}{l}\text { É o gosto amargo que permanece } \\
\text { na boca por um período de tempo } \\
\text { após a ingestão da sobremesa }\end{array}$} & $\begin{array}{l}\text { Nenhum: Chocolate ao leite Nestlé } \\
\text { Forte: Solução café sem açúcar da } \\
\text { marca Nescafé }\end{array}$ \\
\hline \multirow{3}{*}{ Doce } & & Fraco: Leite integral Piracanjuba \\
\hline & \multirow[t]{2}{*}{$\begin{array}{l}\text { Sabor associado a presença de } \\
\text { açucares }\end{array}$} & $\begin{array}{l}\text { Forte: Leite condensado da marca } \\
\text { Nestlé. }\end{array}$ \\
\hline & & Fraco: Chocolate talento vermelho \\
\hline
\end{tabular}


Amendoado

Chocolate
Sabor associado à amêndoa

Sabor associado ao chocolate
Forte: Amêndoa pura

Fraco: achocolatado Muky

Forte: Barra de chocolate $80 \%$ de cacau da marca Cacau Show

\begin{tabular}{|c|c|c|}
\hline Textura & & \\
\hline & & Fraco: Creme de leite Piracanjuba \\
\hline Consistência & $\begin{array}{l}\text { Propriedade de resistência ao } \\
\text { escoamento na boca }\end{array}$ & $\begin{array}{l}\text { Forte: iogurte da marca } \\
\text { Danoninho }\end{array}$ \\
\hline Homogeneidade & $\begin{array}{c}\text { Ausência de grumos ou partículas } \\
\text { que sejam percebidas durante a } \\
\text { deglutição }\end{array}$ & $\begin{array}{l}\text { Fraco: iogurte com } 20 \mathrm{~g} \text { de } \\
\text { granola } \\
\text { Forte: sobremesa láctea Vigor }\end{array}$ \\
\hline
\end{tabular}

Para o treinamento dos julgadores utilizou-se quatro pontos do ensaio do planejamento experimental e na análise sensorial final utilizou-se todos os pontos do planejamento. Após as sessões de treinamento quatro julgadores foram selecionados com base no poder de discriminação e da repetibilidade para compor a equipe sensorial descritiva.

As médias de cada atributo e as respectivas amostras encontradas para a Análise Quantitativa Descritiva são demonstradas na Tabela 2.

Tabela 2 - Médias dos termos descritores das amostras de sobremesa láctea atribuídas pelos assessores pela análise descritiva quantitativa

\begin{tabular}{ccccccc}
\hline Ensaios & \multicolumn{5}{c}{ Atributos } \\
\hline & $\begin{array}{c}\text { Cor } \\
\text { Marrom }\end{array}$ & $\begin{array}{c}\text { Presença } \\
\text { Pe } \\
\text { Partículas }\end{array}$ & Viscosidade & Brilho & $\begin{array}{c}\text { Aroma } \\
\text { Chocolate }\end{array}$ & $\begin{array}{c}\text { Aroma } \\
\text { Amêndoa }\end{array}$ \\
\hline 1 & $1,57^{\mathrm{ac}}$ & $0,71^{\mathrm{b}}$ & $2,28^{\mathrm{b}}$ & $5,43^{\mathrm{bc}}$ & $2,78^{\mathrm{a}}$ & $2,43^{\mathrm{abc}}$ \\
2 & $3,29^{\mathrm{cd}}$ & $7,25^{\mathrm{a}}$ & $4,83^{\mathrm{ab}}$ & $5,18^{\mathrm{bc}}$ & $3,24^{\mathrm{ac}}$ & $6,70^{\mathrm{d}}$ \\
3 & $7,33^{\mathrm{b}}$ & $5,54 \mathrm{a}^{\mathrm{d}}$ & $5,92^{\mathrm{a}}$ & $4,06^{\mathrm{abc}}$ & $6,20^{\mathrm{b}}$ & $4,52^{\mathrm{bcd}}$ \\
4 & $6,08^{\mathrm{b}}$ & $2,36 \mathrm{~b}^{\mathrm{e}}$ & $3,48^{\mathrm{ab}}$ & $4,75^{\mathrm{abc}}$ & $5,80^{\mathrm{bc}}$ & $2,36^{\mathrm{ac}}$ \\
5 & $6,08^{\mathrm{b}}$ & $3,48^{\mathrm{e}}$ & $5,18^{\mathrm{ab}}$ & $5,83^{\mathrm{c}}$ & $5,73^{\mathrm{bc}}$ & $4,15^{\mathrm{bcd}}$ \\
6 & $0,91^{\mathrm{a}}$ & $5,24^{\mathrm{cd}}$ & $3,88^{\mathrm{ab}}$ & $2,02^{\mathrm{a}}$ & $1,82^{\mathrm{a}}$ & $6,09^{\mathrm{d}}$ \\
7 & $6,29^{\mathrm{b}}$ & $5,58^{\mathrm{ad}}$ & $5,72^{\mathrm{a}}$ & $2,81^{\mathrm{ab}}$ & $5,54^{\mathrm{bc}}$ & $5,50^{\mathrm{d}}$ \\
8 & $3,76 \mathrm{~d}$ & $3,85^{\mathrm{cde}}$ & $4,75^{\mathrm{ab}}$ & $3,38^{\mathrm{abc}}$ & $3,83^{\mathrm{abc}}$ & $4,12^{\mathrm{bcd}}$
\end{tabular}




\begin{tabular}{|c|c|c|c|c|c|c|}
\hline 9 & $3,04^{\mathrm{cd}}$ & $3,92^{\text {cde }}$ & $4,44^{\mathrm{ab}}$ & $4,07^{\mathrm{abc}}$ & $3,96^{\mathrm{abc}}$ & $4,18^{\mathrm{bcd}}$ \\
\hline 10 & $3,90^{\mathrm{d}}$ & $3,51^{\mathrm{ce}}$ & $4,43^{\mathrm{ab}}$ & $6,11^{\mathrm{c}}$ & $3,47^{\mathrm{abc}}$ & $4,98^{\mathrm{bd}}$ \\
\hline \multirow[t]{2}{*}{11} & $2,21^{\text {acd }}$ & $0,68^{\mathrm{b}}$ & $2,32^{\mathrm{b}}$ & $5,97^{\mathrm{c}}$ & $3,58^{\mathrm{abc}}$ & $1,16^{\mathrm{a}}$ \\
\hline & $\begin{array}{c}\text { Sabor } \\
\text { Amargo }\end{array}$ & $\begin{array}{l}\text { Sabor } \\
\text { Doce }\end{array}$ & $\begin{array}{c}\text { Sabor } \\
\text { Amendoado }\end{array}$ & $\begin{array}{c}\text { Sabor } \\
\text { Chocolate }\end{array}$ & Consistência & Homogeneidade \\
\hline 1 & $1,99^{\mathrm{ab}}$ & $3,75^{\mathrm{a}}$ & $1,51^{d}$ & $2,36^{\mathrm{bcd}}$ & $0,71^{\mathrm{a}}$ & $7,46^{\mathrm{a}}$ \\
\hline 2 & $2,88^{\mathrm{abcd}}$ & $5,11^{\mathrm{a}}$ & $7,08^{\mathrm{a}}$ & $2,19^{\mathrm{bd}}$ & $5,51^{\mathrm{cd}}$ & $1,67^{\mathrm{b}}$ \\
\hline 3 & $5,48^{c}$ & $4,20^{\mathrm{a}}$ & $5,40^{\text {abe }}$ & $4,62^{\mathrm{acd}}$ & $7,58^{\mathrm{b}}$ & $3,03^{\text {bde }}$ \\
\hline 4 & $4,73^{\mathrm{cd}}$ & $3,62^{\mathrm{a}}$ & $2,13^{\mathrm{cd}}$ & $5,64^{\mathrm{a}}$ & $3,02^{\mathrm{e}}$ & $5,74^{\mathrm{ac}}$ \\
\hline 5 & $4,78^{\mathrm{cd}}$ & $3,67^{\mathrm{a}}$ & $3,13^{\text {cde }}$ & $4,99^{\mathrm{ac}}$ & $6,42^{b}$ & $5,07^{\mathrm{cd}}$ \\
\hline 6 & $2,25^{\mathrm{abd}}$ & $5,04^{\mathrm{a}}$ & $4,63^{\text {be }}$ & $1,59^{\mathrm{b}}$ & $3,33^{\mathrm{e}}$ & $2,94^{\text {be }}$ \\
\hline 7 & $4,12^{\mathrm{abcd}}$ & $4,85^{\mathrm{a}}$ & $6,98^{\mathrm{ab}}$ & $3,60^{\mathrm{abcd}}$ & $7,41^{b}$ & $2,68^{\text {be }}$ \\
\hline 8 & $3,58^{\mathrm{abcd}}$ & $5,13^{\mathrm{a}}$ & $5,49^{\text {abe }}$ & $3,36^{\mathrm{abcd}}$ & $5,52^{\mathrm{cd}}$ & $4,06^{\mathrm{cde}}$ \\
\hline 9 & $4,03^{\mathrm{abcd}}$ & $4,87^{\mathrm{a}}$ & $4,37^{\mathrm{ce}}$ & $4,22^{\mathrm{acd}}$ & $3,51^{\mathrm{e}}$ & $4,23^{\mathrm{cde}}$ \\
\hline 10 & $4,53^{\mathrm{acd}}$ & $3,71^{\mathrm{a}}$ & $3,95^{\mathrm{ce}}$ & $4,61^{\mathrm{abcd}}$ & $4,30^{\mathrm{de}}$ & $4,44^{\text {cde }}$ \\
\hline 11 & $1,57^{\mathrm{b}}$ & $3,28^{\mathrm{a}}$ & $1,05^{\mathrm{d}}$ & $2,38^{\mathrm{bcd}}$ & $0,84^{\mathrm{a}}$ & $7,48^{\mathrm{a}}$ \\
\hline
\end{tabular}

Médias seguidas por letras minúsculas diferentes na mesma coluna indicam que existe diferença significativa a $\mathrm{p}<$ 0,05 pelo teste de tukey

As médias obtidas foram dispostas graficamente no gráfico aranha (Figura 1), traçando o perfil sensorial de cada sobremesa láctea em relação aos atributos estudados, salientando as suas similaridades e diferenças. O centro da figura representa o ponto zero da escala do atributo, e a intensidade aumenta do centro para a periferia da figura. A média de cada atributo é marcada no eixo correspondente e o perfil sensorial traçado pela conexão dos pontos. 


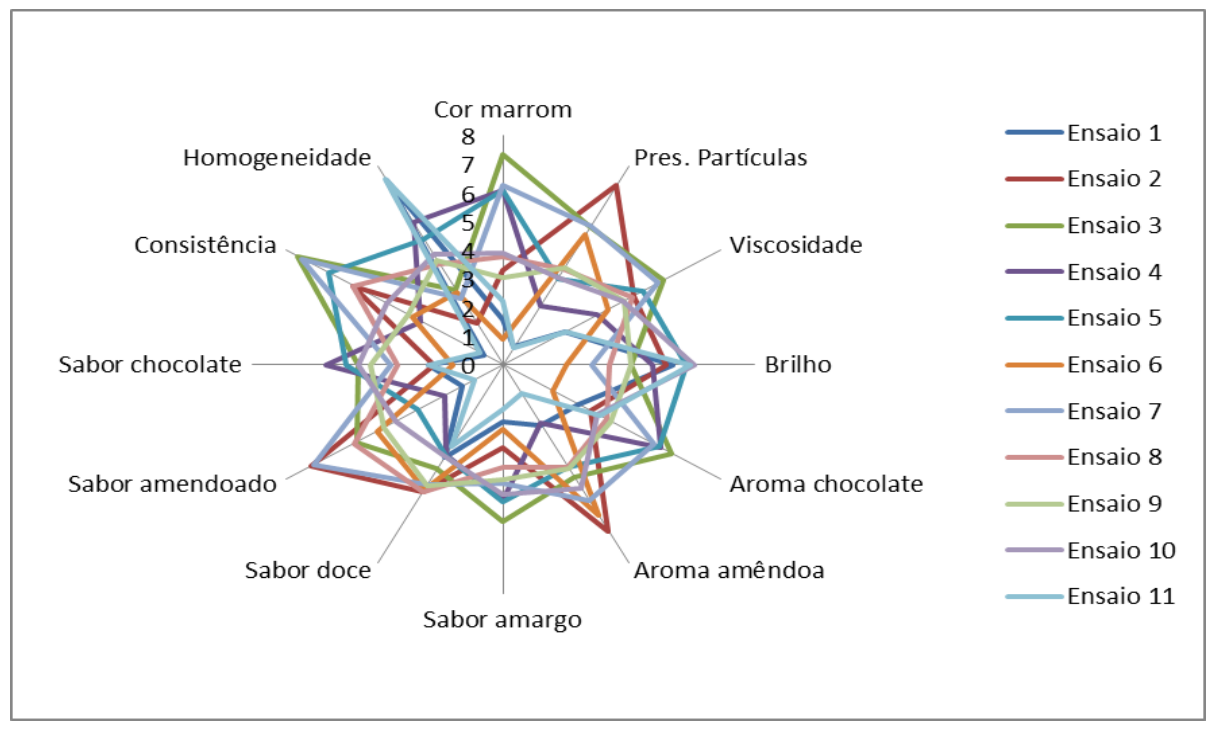

Figura 1 - Gráfico aranha das médias associadas a cada atributo

Observa-se no gráfico que diferentes amostras das sobremesas apresentaram perfis sensoriais diferentes.

Segundo a tabela, os ensaios 3, 7, 4 e 5 apresentaram as maiores médias em relação às demais, não diferindo significativamente entre si quanto ao atributo cor marrom, seguidas pelos ensaios 1, 6 e11 que não apresentaram diferença entre si, sendo o ensaio $6(0,91)$ o que apresentou a menor média.

Os ensaios 1 e 6 também apresentaram menores médias para o aroma chocolate, sabor amargo e sabor chocolate. Estes resultados nos mostram que os atributos estão correlacionados positivamente com a quantidade de chocolate nos ensaios estudados.

Outros atributos relacionados são a presença de partículas, aroma de amêndoa e sabor amendoado. Todos esses foram influenciados pela quantidade da amêndoa do baru nas formulações. As amostras 2 e 7 apresentaram maior intensidade em ambos os atributos não diferindo estatisticamente entre si, já as amostras 11 e 1 apresentaram as menores médias.

Quanto ao atributo viscosidade, a amostra 3 apresentou a maior média diferindo estatisticamente dos ensaios 1 e 11 que apresentaram as menores intensidades do atributo analisado.

As amostras 10 e 11 apresentaram as maiores médias não diferindo estatisticamente entre si para o atributo brilho, porém diferindo estatisticamente da amostra 6 que apresentou a menor média obtendo 2,02de média.

Em relação ao atributo sabor doce, a amostra 8 não diferiu significativamente das demais. Sendo que a maior média foi 5,13 e a menor foi 3,28 da amostra 11.

Por fim, as amostras 7 e 5 apresentaram as maiores médias para o atributo consistência diferindo estatisticamente da amostra 11 que obteve a menor média.

De acordo com os resultados da análise estatística obtidos para os atributos levantados os fatores lineares, quadráticos e de interação não foram significativos a $90 \%$ de confiança, o que 
demonstra que as variáveis independentes chocolate, e baru, não exerceram influência entre os diferentes ensaios.

\subsection{Teste de aceitação e intenção de compra}

A tabela 3 mostra as médias de aceitação dos julgadores para todos os atributos avaliados para sobremesa láctea de chocolate e baru e devido a quantidade de sobremesas foram realizadas análises de somente quatro pontos do planejamento.

Tabela 3 - Médias obtidas para o teste de aceitação de sobremesas lácteas

\begin{tabular}{cccccc}
\hline Ensaios & Cor & Aroma & Textura & Sabor & IC \\
\hline 6 & $8,12^{\mathrm{a}}$ & $8,02^{\mathrm{a}}$ & $7,32^{\mathrm{a}}$ & $7,75^{\mathrm{b}}$ & $4,10^{\mathrm{a}}$ \\
7 & $7,26^{\mathrm{b}}$ & $7,29^{\mathrm{b}}$ & $6,31^{\mathrm{b}}$ & $7,23^{\mathrm{b}}$ & $3,61^{\mathrm{bc}}$ \\
8 & $7,38^{\mathrm{b}}$ & $7,11^{\mathrm{b}}$ & $6,54^{\mathrm{b}}$ & $6,68^{\mathrm{a}}$ & $3,27^{\mathrm{c}}$ \\
9 & $7,46^{\mathrm{b}}$ & $7,39^{\mathrm{b}}$ & $6,81^{\mathrm{ab}}$ & $7,30^{\mathrm{ab}}$ & $3,64^{\mathrm{b}}$ \\
\hline
\end{tabular}

Na mesma coluna, médias com letras em comum não diferem significativamente entre si a $p<0,1$, segundo o teste de Tukey.

Como pode ser observado na tabela 3, os valores obtidos para o atributo cor estão entre 7,26 a 8,12, mostrando que esse atributo foi avaliado pelo julgadores como gostei regularmente a gostei moderadamente. Sendo a formulação 6 com $14 \%$ de chocolate e $7 \%$ de baru a que obteve a maior aceitação pelo atributo cor. Esses valores foram semelhantes aos encontrados por Henrique et al., (2009) no estudo da utilização de maracujá integral no desenvolvimento de sobremesa láctea que obtiveram valores entre 7,85 a 7,98 para esse atributo.

Para o aroma, as médias variaram de 7,11 a 8,0 e a amostra 6 foi a que obteve maior aceitação com média de 8,02. Esses valores encontrados foram semelhantes aos 7,38 e 7,68 encontrados por silva et al., (2012) no estudo da avaliação de parâmetros sensoriais em sobremesas lácteas sabor chocolate elaboradas com leite e soro de leite.

Em relação à textura as maiores médias foram das formulações $6(7,32)$ e $9(6,81)$ não diferindo estatisticamente entre si, seguidas pelas formulações 8 e 7 que obteve as médias 6,54 e 6,31 respectivamente também não diferindo estatisticamente entre si. Os valores encontrados foram levemente inferiores aos 6,9; 7,0 e 7,2 encontrados por Salviano et al., (2012) no estudo de desenvolvimento e aceitabilidade de sobremesa fermentada caprina sabor manga.

Os ensaios apresentaram média de aceitação para o sabor entre 6,68 a 7,75, avaliando esse atributo como gostei ligeiramente a gostei regularmente. A menor média foi da formulação 8 $(6,68)$ que é o ponto máximo de baru $(14 \%)$ e o ponto central de chocolate $(9 \%)$ e a maior média foi novamente da formulação 6 que contém o ponto máximo de chocolate (14\%) e o ponto central de baru (7\%). Esses valores foram semelhantes aos 7,1; 7,1 e 6,9 encontrados por Salviano et al., (2012) no estudo de desenvolvimento e aceitabilidade de sobremesa fermentada caprina sabor manga.

Para o atributo intenção de compra as médias variaram de 3,27 a 4,10 avaliando a intenção dos julgadores como talvez compraria / talvez não compraria a provavelmente compraria. A maior média foi da amostra $6(4,10)$ diferindo significativamente da amostra $8(3,27)$ que obteve a menor média. 


\section{CONCLUSÃO}

A ADQ mostrou que existe uma grande heterogeneidade nos termos descritores na qualidade sensorial das sobremesas lácteas produzidas e a concentração de chocolate e baru não foram significativas, mostrando que as varivéis não influenciaram nas características sensoriais das diferentes formulações.

$\mathrm{Na}$ análise de aceitação e intenção de compra o ensaio 6 (14 de chocolate e 7\% de baru) destacou-se pela cor, aroma, textura e maior intenção de compra pelos consumidores.

\section{REFERÊNCIAS}

DAMÁSIO, M. H.; COSTELL, E. Análisis sensorial descriptivo: Generación de descriptores y selección de catadores. Agroquímica Tecnol Alimentos., v.31/2, p.165-78, 1991.

HENRIQUE, J. R. et al. Utilização de maracujá integral no desenvolvimento de sobremesa láctea (flan) e avaliação de suas características físico-químicas e sensorial. In: II Semana de Ciência e Tecnologia do IFMG, 2009, Bambuí, MG, pg. 1-5.

LIMA, J. C. R. et al. Qualidade microbiológica, aceitabilidade e valor nutricional de barras de cereais formuladas com polpa e mêndoa de baru. Boletim do Centro de Pesquisa e Processamento de Alimentos. Curitiba, v. 28, n. 2, p.331-343, 2010.

SALVIANO, A, T, M; et al. Desenvolvimento e aceitabilidade de sobremesa fermentada caprina sabor manga. Revista Brasileira de Produtos Agroindustriais, Campina Grande, v.14, n.2, p.185190, 2012.

SILVA, A, S; et al. Avaliação de parâmetros sensoriais em sobremesas lácteas sabor chocolate elaboradas com leite e soro de leite. In: Congresso Norte Nordeste de pesquisa e inovação, Palmas, TO, 2012.

STONE, H.; BLEIBAUM, R.N.; THOMAS, H.A. Sensory evaluation practices. 4. ed., New York: Academic Press. 2012. 438p.

SOLER, N. et al. elaboração, composição química e avaliação sensorial de sobremesas lácteas achocolatadas com abacate. Alim. Nutr., Araraquara, v. 22, n. 1, p. 143-148, jan./mar, 2011.

TEIXEIRA, E.; MEINERT, E.; BARBETTA, P. A. Análise sensorial dos alimentos. Florianópolis: Ed. UFSC, 1987. p. 182. 